\title{
Real-Time Support in the Proposal for Fine-Grained Parallelism in Ada
}

\section{Wortvarton}

$\square$ Real-time parallel models are now common

$\square$ But little exists on fine-grained parallelism within real-time languages and runtimes

$\square$ Ada is a language of choice for reliable real-time systems

$\square$ It incorporates models of computation which are amenable for real-time analysis

- Ada 2012 supports many real-time multiprocessor scheduling schemes, global and partitioned

Existent multiprocessor support in Ada follows a coarse-grained model

$\square$ It needs to be augmented with lightweight fine-grained parallelism. 


\section{Luís Miguel Pinho} CISTER (Portugal)

\author{
Stephen Michell
}

Maurya Software Inc (Canada)

\section{Brad Moore}

General Dynamics (Canada)
S. Tucker Taft AdaCore (USA)

\section{Ada 2022x ftine-gialined] [parallellism}

$\square$ Definition of a parallel non-schedulable unit (Tasklet)

$\square$ Explicit or implicit parallelization

$\square$ Ada Tasks execute graphs of Tasklets
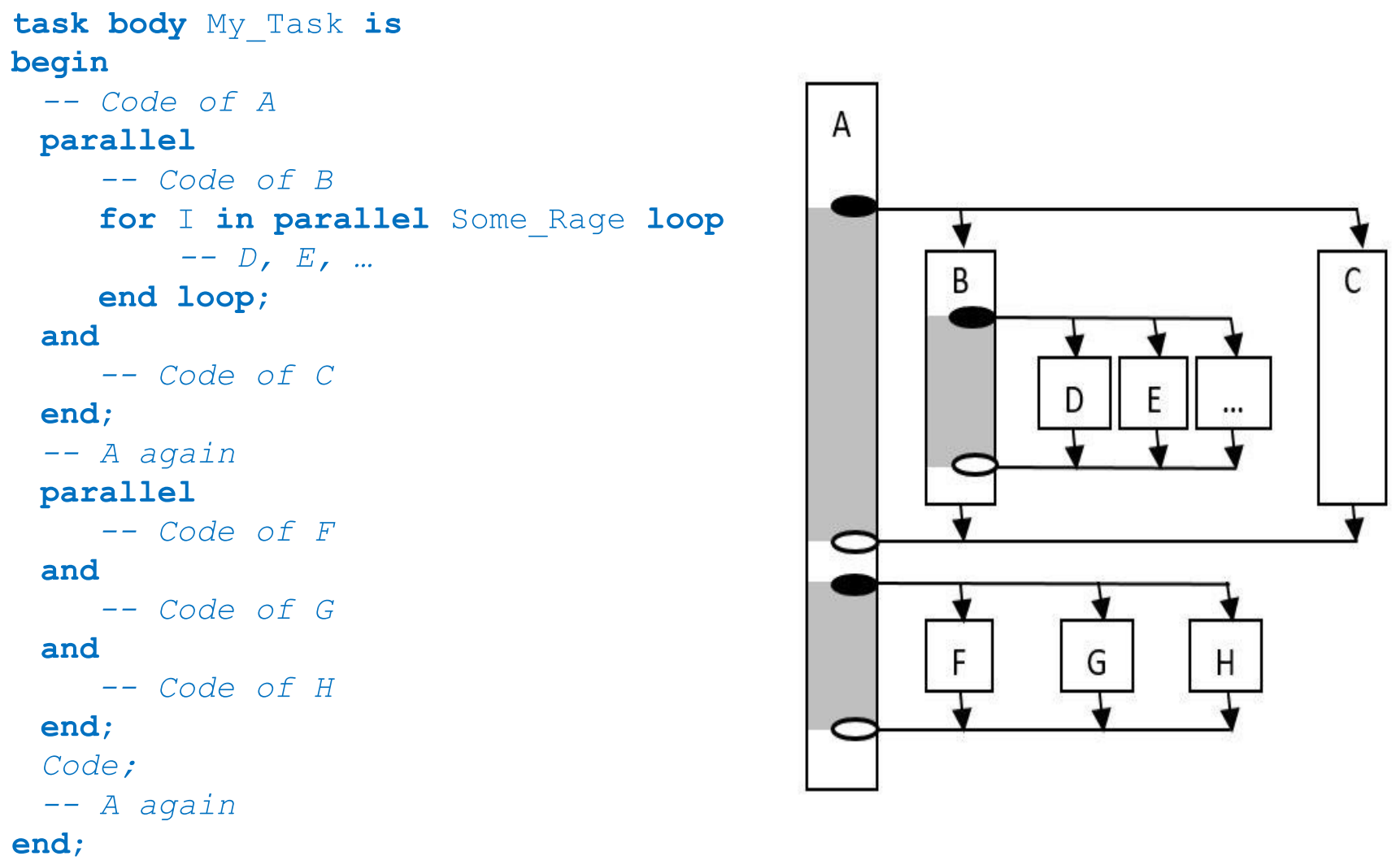


\section{Exఆcurton MTode]}

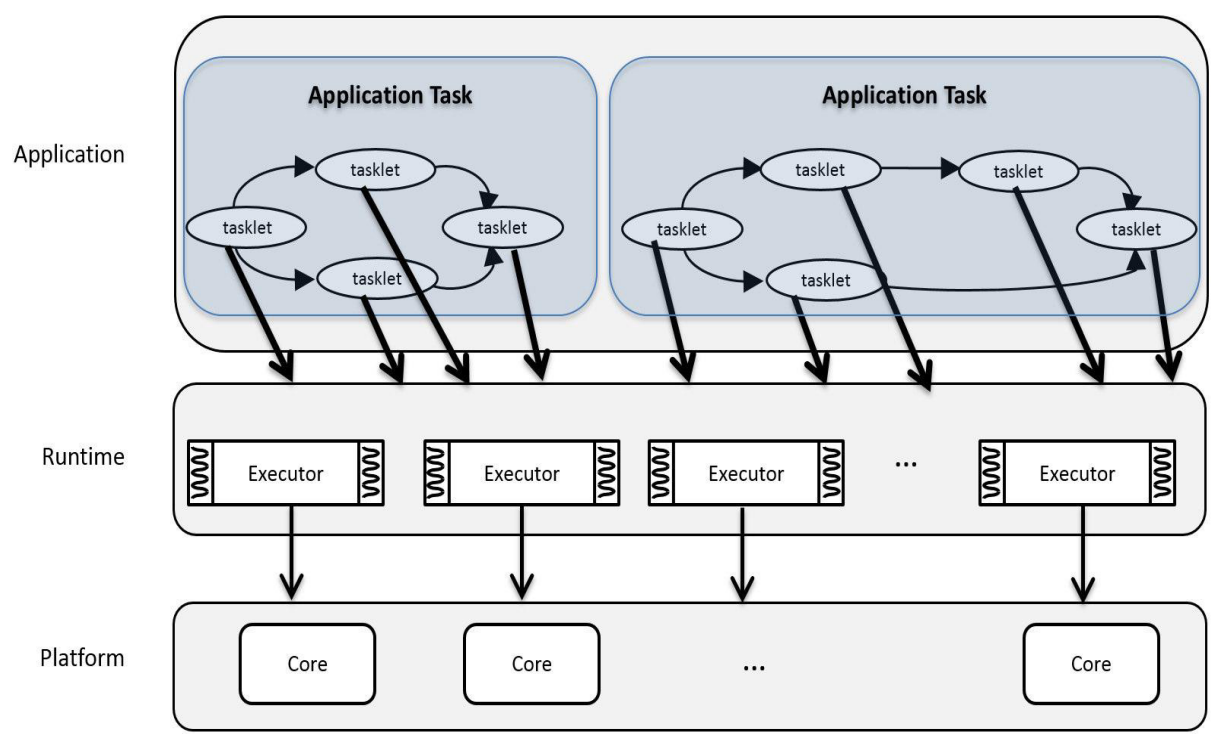

$\square$ Tasklets are executed by Executors

$\square$ E.g. OS threads, but can be bare metal entities

$\square$ Limited form of run-to-completion

A tasklet is mapped to one executor, except if blocking

$\square$ Executor might be scheduled in a preemptive, global or partitioned scheduling

$\square$ Allocation of tasklets to executors, and of executors to cores is left to the implementation

$\square$ Models are defined to guarantee safeness and progress, even with potential blocking operations 


\section{Realltime lissues}

$\square$ Each Ada task (or priority) is provided with a specific executor pool

$\square$ All executors carry the same priority/deadline of the task

$\square$ Does not support graph decomposition techniques

Maps to a synchronous fork-join model

$\square$ for which analysis already exists
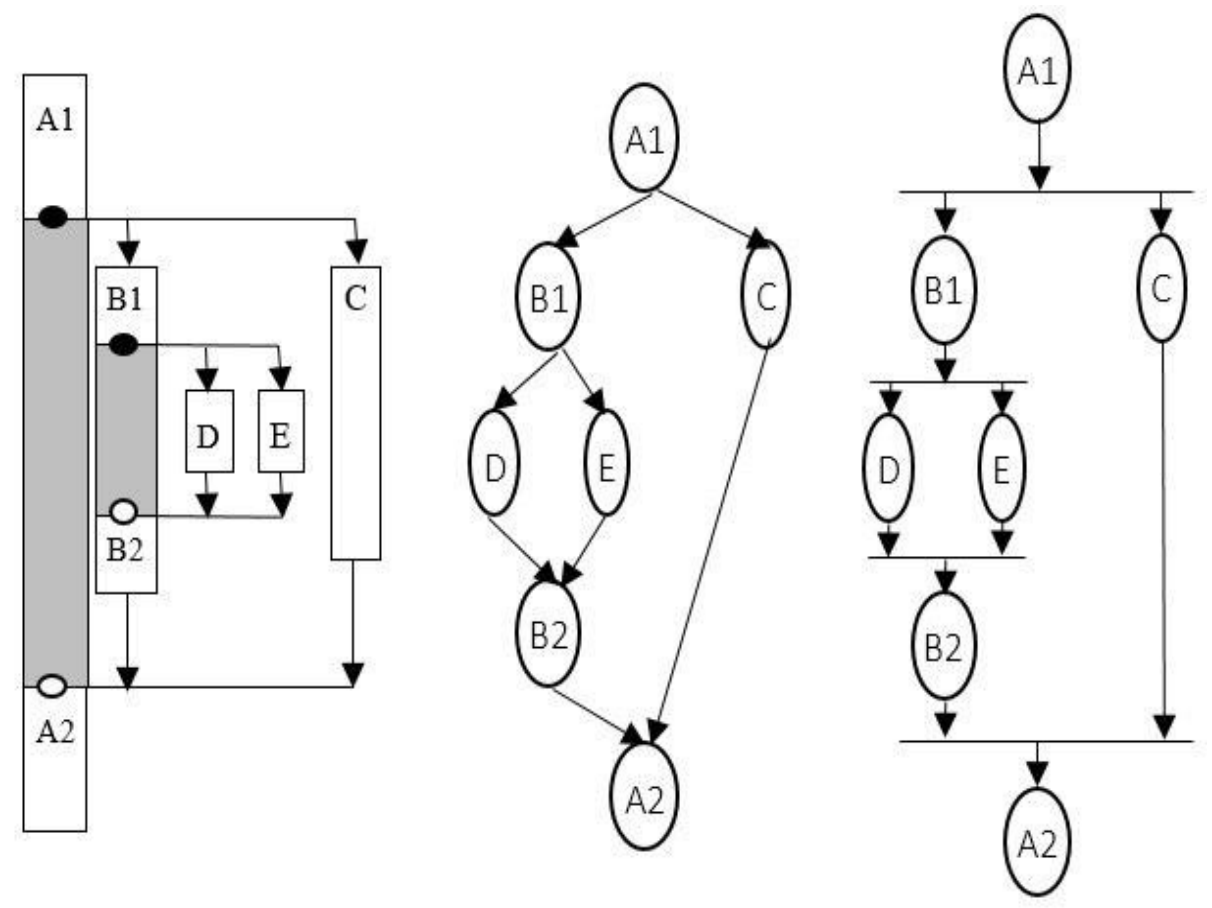

$\square$ Important open issues

- Supported scheduling models, such as limited preemption

Run-to-completion and tasklet (work-)stealing

- Parallel models in languages introduce additional issues $\square$ (e.g. integration with task abortion, exceptions, etc.) 